\title{
Host hybridization as a potential mechanism of lateral symbiont transfer in deep-sea vesicomyid clams
}

\author{
Corinna Breusing $^{1,2}$ (i) | Shannon B. Johnson ${ }^{1}$ | Robert C. Vrijenhoek ${ }^{1}$ | Curtis R. Young ${ }^{2}$
}

\author{
${ }^{1}$ Monterey Bay Aquarium Research Institute, \\ Moss Landing, CA, USA \\ ${ }^{2}$ National Oceanography Centre, \\ Southampton, UK \\ Correspondence \\ Corinna Breusing, Graduate School of \\ Oceanography, University of Rhode Island, \\ 215 S Ferry Rd, Narragansett, RI 02882, \\ USA. \\ Email: corinnabreusing@gmail.com \\ Funding information \\ David and Lucile Packard Foundation; \\ Natural Environment Research Council, \\ Grant/Award Number: NE/N006496/1 \\ and NE/R015953/1; Deutsche \\ Forschungsgemeinschaft, Grant/Award \\ Number: BR 5488/1-1; National Science \\ Foundation, Grant/Award Number: \\ OCE0241613 and OCE9302205
}

\begin{abstract}
Deep-sea vesicomyid clams live in mutualistic symbiosis with chemosynthetic bacteria that are inherited through the maternal germ line. On evolutionary timescales, strictly vertical transmission should lead to cospeciation of host mitochondrial and symbiont lineages; nonetheless, examples of incongruent phylogenies have been reported, suggesting that symbionts are occasionally horizontally transmitted between host species. The current paradigm for vesicomyid clams holds that direct transfers cause host shifts or mixtures of symbionts. An alternative hypothesis suggests that hybridization between host species might explain symbiont transfers. Two clam species, Archivesica gigas and Phreagena soyoae, frequently co-occur at deep-sea hydrocarbon seeps in the eastern Pacific Ocean. Although the two species typically host gammaproteobacterial symbiont lineages marked by divergent $16 \mathrm{~S}$ rRNA phylotypes, we identified a number of clams with the $A$. gigas mitotype that hosted symbionts with the $P$. soyoae phylotype. Demographic inference models based on genome-wide SNP data and three Sanger sequenced gene markers provided evidence that $A$. gigas and $P$. soyoae hybridized in the past, supporting the hypothesis that hybridization might be a viable mechanism of interspecific symbiont transfer. These findings provide new perspectives on the evolution of vertically transmitted symbionts and their hosts in deep-sea chemosynthetic environments.
\end{abstract}

\section{KEYWORDS}

horizontal transfer, hybridization, symbiosis, vertical transmission, vesicomyid clams

\section{1 | INTRODUCTION}

Host-microbe symbioses are universal phenomena that are now considered key drivers of evolutionary innovation (Archibald, 2015; Brucker \& Bordenstein, 2012; McFall-Ngai, 2008; McFall-Ngai et al., 2013). Over the past several decades, it has been established that symbiotic associations led to the evolution of cellular organelles and eukaryotic cell life (Archibald, 2015), while recent studies have emphasized their role in the formation of species (Brucker \& Bordenstein, 2012). Transmission of microbes contributes to maintenance of symbiotic relationships across host generations and differences in transmission modes have important implications for the evolution of both partners (Bright \& Bulgheresi, 2013; Vrijenhoek, 2010). Under vertical transmission, symbionts are inherited through the maternal and/or-less frequently-the paternal germ line (e.g., Ebert, 2013; Moran \& Dunbar, 2006; Watanabe, Yukuhiro, Matsuura, Fukatsu, \& Noda, 2014). In the predominant case of uniparental maternal inheritance, symbiont and mitochondrial genomes are cotransmitted and are thus genetically and evolutionarily linked. Bottleneck effects during transovarial transmission 
strongly reduce the effective size and genetic diversity of the symbiont population within individual hosts, thereby increasing the fixation of slightly deleterious mutations (Rispe \& Moran, 2000; Vrijenhoek, 2010). Since recombination with environmental bacteria is limited and certain symbiont gene functions become obsolete or are complemented by the host or secondary symbiotic microbes, vertically transmitted symbionts typically lose genes through drift and selection, resulting in significant reductions in genome size (Bennett \& Moran, 2015; Moran, McCutcheon, \& Nakabachi, 2008; Sloan \& Moran, 2012). Apart from vertical transmission, symbionts can be transmitted horizontally, either through uptake of free-living strains in the environment or through direct transfer between hosts (Bright \& Bulgheresi, 2013; Ebert, 2013; Vrijenhoek, 2010). Because symbionts are acquired from a potentially diverse mixture of bacterial strains each generation, horizontal transmission often results in genetic heterogeneity in the symbiont population and the absence of co-evolution between host and symbiont. In contrast to vertically transmitted symbionts, horizontally transmitted symbionts switch between intra- and extrahost life phases, which increases rates of recombination and selective pressures for retaining genes necessary for surviving outside the host environment (Vrijenhoek, 2010). In various cases, it has been shown that horizontal transmission can supplement the vertical transmission mode (Ebert, 2013), thereby providing opportunities for recombination that can counteract the ongoing genome degradation in vertically transmitted symbionts (Vrijenhoek, 2010). Despite growing research on diverse host-microbe relationships, the mechanisms of symbiont transmission and their evolutionary consequences are still poorly understood (Bright \& Bulgheresi, 2013).

Deep-sea invertebrates that inhabit hydrothermal vents, hydrocarbon seeps and sites of organic enrichment have evolved intriguing symbioses that compensate for the absence of sunlight and the trophic benefits of photosynthesis. Associations with chemoautotrophic bacteria that derive energy from the oxidation of sulphides, hydrogen or methane can support lush invertebrate communities in these unusual habitats (Dubilier, Bergin, \& Lott, 2008). Clams of the family Vesicomyidae belong to the key fauna in chemosynthesis-based ecosystems worldwide (Johnson, Krylova, Audzijonyte, Sahling, \& Vrijenhoek, 2016). Lacking a functional digestive system, they rely nutritionally on their thiotrophic gammaproteobacterial symbionts that inhabit specialized cells in the gill tissue of their host. Previous histological and molecular studies showed that vesicomyid symbionts are maternally inherited and can be grouped into two different phylogenetic clades that differ in their status of genome reduction (Kuwahara et al., 2011). Clade I symbionts have highly reduced genomes that lack crucial genes for DNA recombination and repair, whereas clade II symbionts retain functional copies of these genes and have slightly larger genome sizes (Kuwahara et al., 2007, 2011; Shimamura et al., 2017).

Although maternal inheritance appears to be the main transmission route of these symbionts, rare occasions of horizontal transfer have been suggested given that host mitochondrial and symbiont $16 \mathrm{~S}$ rRNA phylogenies are sometimes incongruent (Ozawa et al., 2017; Stewart, Young, \& Cavanaugh, 2008, 2009; Vrijenhoek, 2010). Different mechanisms have been proposed to explain how lateral acquisition of symbionts could occur in vesicomyid clams (Stewart, Young, \& Cavanaugh, 2008): (a) hybridization between host species including the presence of doubly uniparental inheritance, (b) acquisition from a stable free-living symbiont population, (c) direct transfer between hosts without the involvement of hybridization, for example through contact between eggs, contact between eggs and host tissue or uptake of symbionts that have been released from moribund clams.

Two recent studies hypothesized that direct transfer is the main mechanism leading to symbiont mixtures or displacements of native symbionts in vesicomyid clams. Decker, Olu, Arnaud-Haond, and Duperron (2013) reported that individual vesicomyid clams from the Gulf of Guinea can host mixtures of native and non-native symbiont phylotypes when distinct host species co-occur in the same seep habitat. These authors argued that physical proximity could promote symbiont exchanges among very distantly related clam taxa. Ikuta et al. (2016) recently showed that the symbiont of Phreagena okutanii (previously Calyptogena okutanii) spends part of its life attached to the surface of the host's eggs, thereby strengthening Stewart et al.'s (2008) argument that direct contact between eggs or eggs and host tissues can lead to lateral symbiont transfer between co-occurring clam species. While these studies considered the possibility of host hybridization or environmental symbiont acquisition unlikely, these hypotheses have not been directly addressed by previous analyses. Here, we present a new case of discrepant symbiont compositions in two eastern Pacific clams, Archivesica gigas and Phreagena soyoae, species that are easily distinguished based on mitochondrial and nuclear markers (Johnson et al., 2016). Using demographic inference models based on genome-wide SNPs as well as traditional DNA markers, we investigated the hypothesis that hybridization between the two species might be a mechanism of horizontal symbiont transfer in this system and examined the nature of this gene flow between the taxa.

\section{2 | MATERIAL AND METHODS}

\section{1 | Sample collection and preparation}

Clams were collected with remotely operated vehicles (ROVs) from eight eastern Pacific seep sites during cruises between 2000 and 2015 (Table 1; Figure 1). Upon recovery of the ROV, specimens were either dissected and frozen at $-80^{\circ} \mathrm{C}$ or preserved in $95 \%$ ethanol. DNA was extracted from symbiont-bearing gill and symbiont-free foot or adductor muscle tissue with the QIAGEN DNeasy Blood \& Tissue Kit according to manufacturer's instructions. An RNA digestion step was included as advised in the protocol. We constructed the map of sampling localities with GGMAP in RSTUDIO (https://cran.r-project.org/web/packages/ggmap/citat ion.html). 
TAB LE 1 Geographic coordinates, depths, dive numbers, sample sizes $(N)$ and host species for the investigated clam sites

\begin{tabular}{|c|c|c|c|c|c|c|c|}
\hline Locality & Lat & Lon & Depth (m) & Dive $^{a}$ & $N$ & Year & Host $^{t}$ \\
\hline Coronado Canyon & 32.36 & -117.38 & 1,266 & D766 & 2 & 2015 & PA \\
\hline San Diego Fault & 32.91 & -117.77 & 999 & D625 & 5 & 2014 & $\mathrm{PA}$ \\
\hline Pedro's Whalefall & 33.77 & -119.52 & 1895 & D464, D474 & 14 & 2013 & A \\
\hline Extrovert Cliff & 36.77 & -122.08 & 960 & $\begin{array}{l}\text { V1676, V1682 } \\
\text { V2034, T233 }\end{array}$ & 28 & 2000-2001 & PA \\
\hline Gorda Ridge & 40.36 & -125.21 & 1588 & T349 & 10 & 2001 & $A$ \\
\hline
\end{tabular}

a Submersibles: $\mathrm{D}=$ Doc Ricketts, $\mathrm{T}=$ Tiburon, $\mathrm{V}=$ Ventana.

${ }^{\mathrm{b}}$ Host: $\mathrm{P}=$ Phreagena soyoae, $\mathrm{A}=$ Archivesica gigas.

\section{2 | Sanger sequencing of host and symbiont genes}

The mitochondrial cytochrome c oxidase subunit I (COI), the nuclear histone $3(\mathrm{H} 3)$ and the nuclear ADP/ATP translocase (ANT) genes were used for host species identification. PCR and sequencing protocols followed Johnson et al. (2016). Assembly of forward and reverse reads, multiple alignments and phasing of nuclear genes were done as in Breusing, Johnson, Tunnicliffe, and Vrijenhoek (2015). To identify the dominant symbiont lineage in the sampled clam species, we sequenced the full-length $16 \mathrm{~S}$ rRNA using the universal eubacterial primers 27F/1492R (Lane, 1991). Gene amplifications and sequencing reactions were performed as in Vrijenhoek, Duhaime, and Jones (2007), while sequence analysis was done as described above.

\section{3 | ezRAD sequencing and estimation of allele frequencies}

The Sanger sequence analyses indicated that several clams with the A. gigas $\mathrm{COI}$ mitotype contained the $P$. soyoae symbiont $16 \mathrm{~S}$ phylotype. To determine whether hybridization had occurred between the two host species and could therefore possibly account for the observed symbiont switch, we developed a SNP panel from ezRAD sequencing of five putatively pure A. gigas (Gorda Ridge) and five putatively pure $P$. soyoae (Clam Bed) individuals. These sites were chosen as references as they each contained only one clam species without any evidence for genetic admixture or symbiont discrepancies. The composition of these SNPs was evaluated in four clams from Pedro's Whalefall in which host-symbiont discrepancies were found. Library preparation and sequencing was performed at the Huntsman Cancer Institute at the University of Utah and UC Davis. The library preparation protocol was adapted from the original methods described in Toonen et al. (2013) and is provided in full detail in Appendix S1. Sequencing of the 14 clams was done with a $2 \times 125-150$ bp paired-end protocol on Illumina HiSeq 2500 and 4000 instruments. Following quality checks with FASTQC (https://www.bioinformatics.babraham.ac.uk/projects/fastq $\mathrm{c} /$ ), the raw reads were compared against draft genome assemblies of the clam symbionts and host mitochondrial and ribosomal genes (C. R. Young, unpublished data) as well as the PhiX sequencing control to remove potential contaminants and obtain a purely nuclear gene data set (Appendix S2). Unmapped paired-end reads were then trimmed and quality filtered with tRIMmOMATIC (Bolger, Lohse, \& Usadel, 2014) and assembled in DDOCENT version 2.2.17 (Puritz, Hollenbeck, \& Gold, 2014) following recommendations for assembly optimization at dDocent.com. Assembly parameters were adjusted as follows: clustering threshold $=0.9$; minimum coverage of a read within an individual $=6$; minimum number of individuals containing a unique sequence $=4$. Basic quality metrics and information about the sequencing data are given in Appendix S3. Exhaustive exploration of various settings in the DDOCENT SNP filtering pipeline all resulted in spurious patterns in population-specific allele frequency spectra (AFS) and poor convergence in downstream population genomic analyses. As recently shown by Warmuth and Ellegren (2019), traditional SNP calling from RADseq data can introduce bias in demographic inference. Based on these results, we used ANGSD version 0.920 (Korneliussen, Albrechtsen, \& Nielsen, 2014) to estimate AFSs and other population genetic statistics in this study (Appendix S4). To remove low-quality sites from the analyses, we used a minimum mapping quality of 30 ( $\operatorname{minMapQ}=30$ ), a minimum base quality of $20(\mathrm{minQ}=20)$ and a minimum depth of 20 (setMinDepth $=20$ ). We further adjusted mapping quality for excessive mismatches $(C=50)$, removed sites with missing data, excluded spurious and improperly paired reads and computed per-base alignment qualities $(B A Q=1)$ to resolve false variants that were caused by misalignments. Potentially paralogous regions were excluded by discarding reads that had multiple hits to the reference assembly and by considering only sites that had a maximum depth of 250 (which we chose as reasonable threshold based on the mean read depth distribution). Inferences were based on the folded AFS due to no outgroup information available before the analysis. The joint AFS between A. gigas and $P$. soyoae was calculated with the ANGSD subprogram REALSFS and subsequently folded in $\partial_{A} \partial$ I version 1.6.3 (Gutenkunst, Hernandez, Williamson, \& Bustamante, 2009).

\subsection{Phylogenetic and population genetic analyses}

We used the program POPART version 1.7 (http://popart.otago. ac.nz/) to create phylogenetic networks for the symbiont 16 S rRNA 


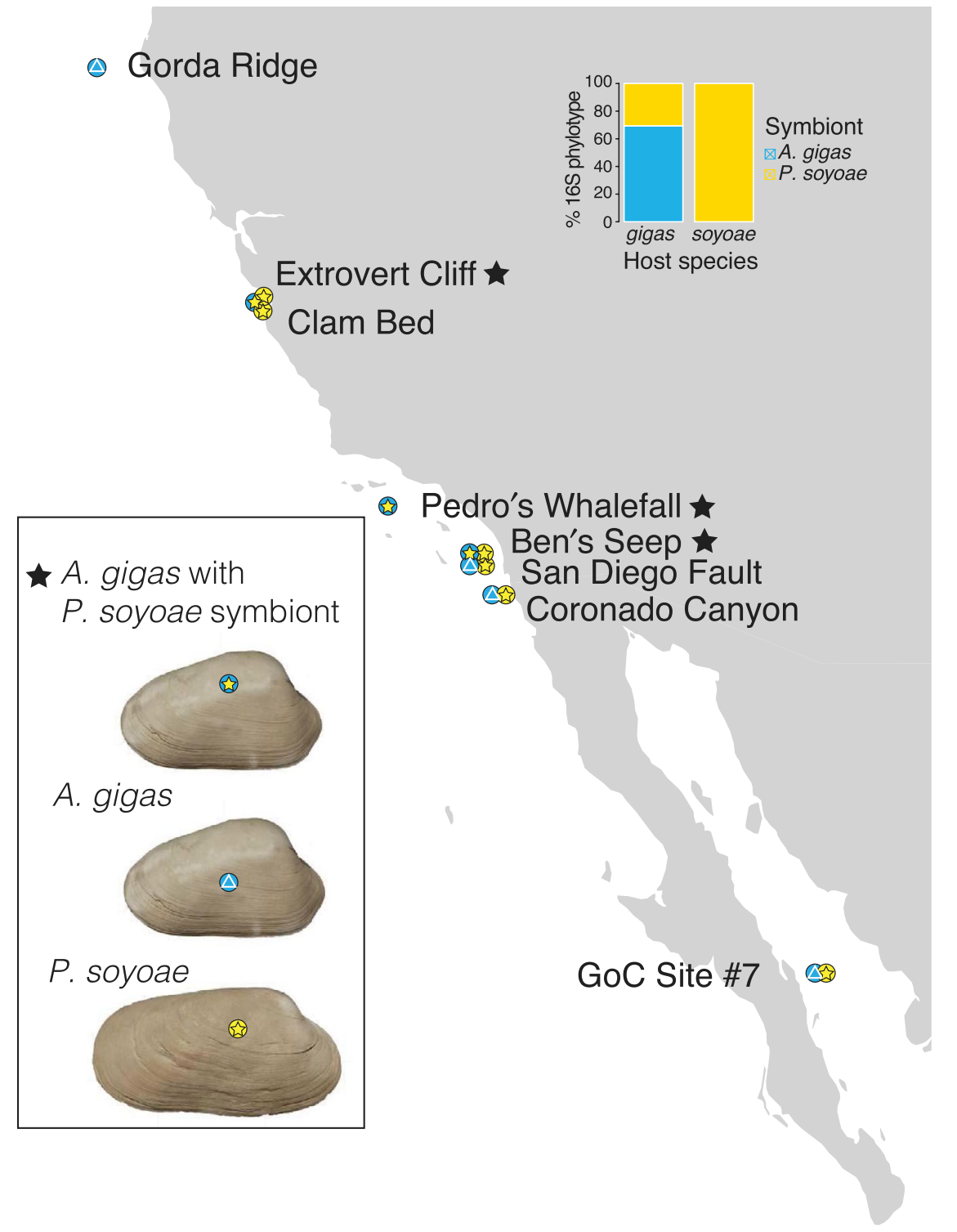

FIGURE 1 Sampling sites for clam specimens in the eastern Pacific Ocean. Blue circle $=$ A. gigas host, yellow circle $=P$. soyoae host, blue triangle $=$ A. gigas symbiont, yellow star $=P$. soyoae symbiont. The barplot in the upper right shows the proportion of each symbiont type in the two host species across all individuals sampled. Clam images were taken by Shannon Johnson

gene and the host mitochondrial and nuclear genes. Networks were generated based on the median-joining algorithm with the epsilon parameter set to 0 . Diversity and $F_{\mathrm{ST}}$ statistics for Sanger data were calculated in MEGA version 10.0.5 (Kumar, Stecher, Li, Knyaz, \& Tamura, 2018) and GeNODIVE version 2.0b27 (Meirmans $\&$ van Tienderen, 2004), respectively. $F_{\mathrm{ST}}$ values were corrected after Benjamini and Yekutieli (2001). To obtain estimates of genomic divergence and structure, we computed pairwise $F_{S T} S$, PCAs and admixture proportions from the RADseq data set using the NGSTOOLS version 1.0.2 package (Fumagalli, Vieira, Linderoth, \& Nielsen, 2014) and the NGSADMIX subprogram with 100,000 maximum iterations in ANGSD.

\section{5 | Demographic inference}

We used the program IMA2 (Hey, 2010) on the three Sanger sequenced genes mtCOI, $\mathrm{H} 3$ and ANT to test whether introgression had occurred in the evolutionary history of the two clam species or whether shared polymorphisms were mostly a result of incomplete lineage sorting. IMA2 was run under a two-population model differentiated by $A$. gigas and $P$. soyoae genotypes. Isolation-with-migration analyses make several assumptions about the nature of the data, including no recombination within genes, no genetic linkage, absence of population structure and gene flow from unsampled species as well as selective neutrality. While most assumptions are robust to moderate levels of violation (Strasburg \& Rieseberg, 2010), recombination can introduce significant bias into parameter estimates. To exclude recombining fragments from the analyses, we applied the four-gamete test in the program IMGC (Woerner, Cox, \& Hammer, 2007). The infinite sites substitution model with an inheritance scalar of 1.0 was used for all nuclear genes and 0.25 was used for the mitochondrial $\mathrm{COI}$ locus under the HKY model. Analyses were run multiple times with at least $10^{7}$ steps, where the first $10^{4}$ steps were discarded as a burn-in. We used geometric heating with parameters between 0.99 and 0.3 with 50 attempts at chain swapping per iteration between the $50-80$ chains. 
FIGURE 2 Haplotype network for host (mtCOI, ANT, H3) and symbiont (sym16S) genes. Each circle represents a single haplotype where circle size is proportional to frequency in the data set. Lines on connecting branches indicate number of mutations between haplotypes. For ANT, $\mathrm{H} 3$ and sym16S, P. soyoae-specific alleles (yellow) are found in A. gigas (blue)

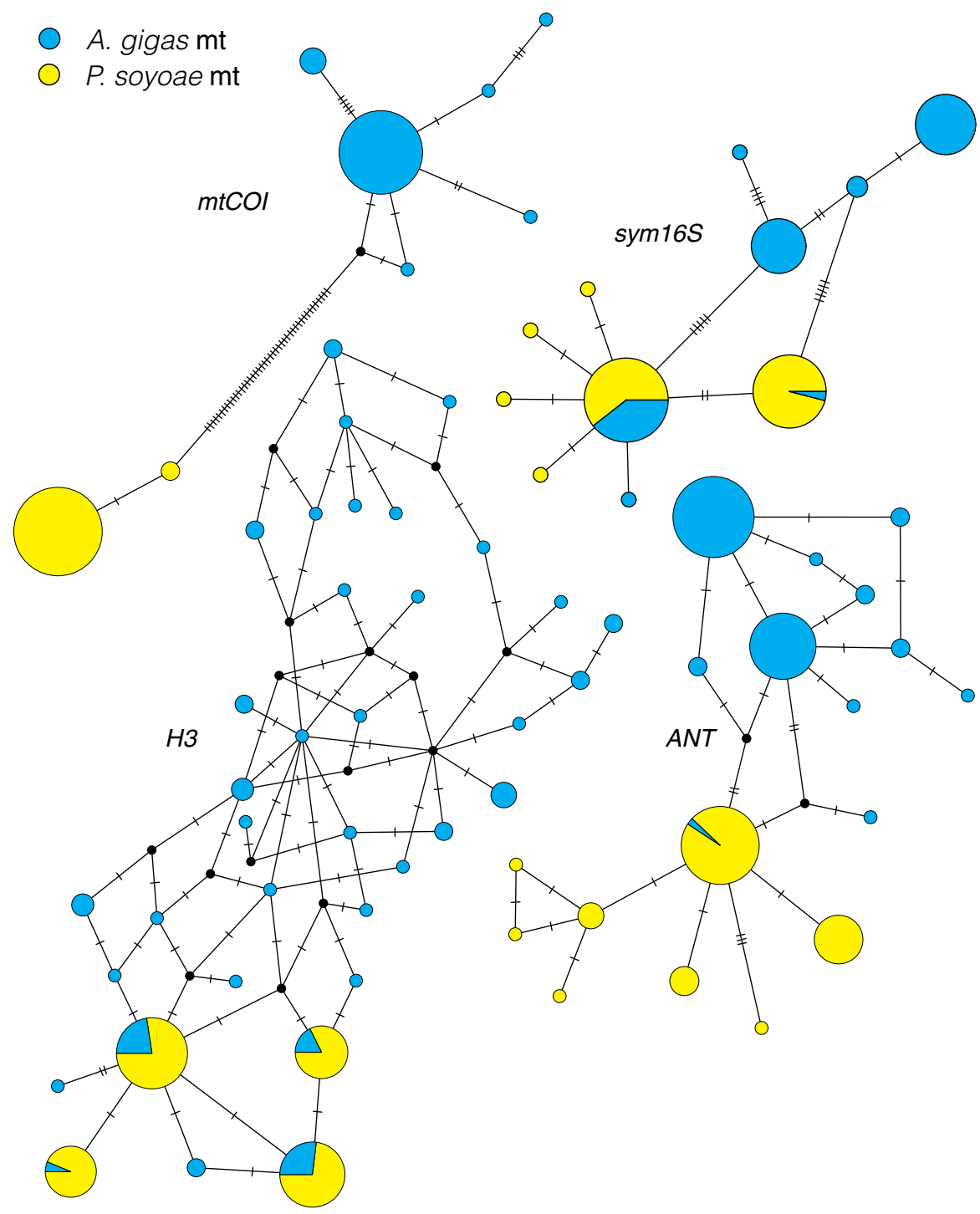

Complementary to the IMA2 approach, the program $\partial_{A} \partial$ I version 1.6.3 (Gutenkunst et al., 2009) was used to infer the demographic history of the two clam species from the folded joint allele frequency spectrum estimated from the RADseq data set. This approach is considerably more flexible than IMA2 with respect to demographic models and the variability of rates among different genomic regions. As in the IMA2, we defined two populations based on the respective genotypic signature. We tested seven different models of evolutionary divergence as implemented in Tine et al. (2014): strict isolation (SI), isolation-with-migration (IM), ancient migration (AM), secondary contact (SC), as well as IM, AM and SC with heterogeneous introgression across genomic loci (IM2M, AM2M, SC2M). All models were fitted using hot and cold annealing followed by L-BFGS-B optimization. For each model, we performed 80 independent runs with 5,000 iterations per optimization to find the global maximum. After excluding spurious runs where parameter estimates hit the model boundaries, the iteration with the lowest Akaike information criterion (AIC) was chosen as best fit. AIC weights (Burnham \& Anderson, 2002; Stewart et al., 2008) were used to express relative support among the set of models that we examined.

\section{3 | RESULTS}

\section{1 | Sanger sequenced genes: Haplotype diversity and differentiation in hosts and symbionts}

Phylogenetic networks for a 518-bp fragment of the host mitochondrial $\mathrm{COI}$ gene revealed a clear segregation of haplotypes into A. gigas- and P. soyoae-specific clades (Figure 2). Haplotypes for this gene were the most divergent and were fixed between the two clam species (9.4\% K2P distance). Within-clade divergence and overall haplotype diversity $(H)$ were low $(A$. gigas: $0.02 \% \mathrm{~K} 2 \mathrm{P}$; P. soyoae: $0.21 \% \mathrm{~K} 2 \mathrm{P}$; eight haplotypes; $H=0.6 \pm 0.03 \mathrm{SD}$ ). Based on $\mathrm{mtCO}$, the $A$. gigas and $P$. soyoae populations were strongly differentiated from each other $\left(F_{\mathrm{ST}}: 0.561-1.000\right)$, while no population differentiation was observed within species (Appendix S5). Although the nuclear genes ANT and $\mathrm{H} 3$ could be grouped into $A$. gigas- and $P$. soyoae-specific clades as well, some haplotypes were shared between species. In both cases, A. gigas contained polymorphisms that were characteristic of $P$. soyoae, whereas the opposite case was not observed. Compared to $\mathrm{mtCOI}$, the between-clade sequence 
divergence estimates for both nuclear genes were lower $(0.89 \% \mathrm{~K} 2 \mathrm{P}$ for ANT and $1.1 \% \mathrm{~K} 2 \mathrm{P}$ for $\mathrm{H} 3$ ), while the within-clade sequence divergence and haplotype diversity were higher (ANT: 0.21\% K2P for A. gigas and $0.20 \% \mathrm{~K} 2 \mathrm{P}$ for $\mathrm{P}$. soyoae; $\mathrm{H}=0.81 \pm 0.0017 \mathrm{SD} ; \mathrm{H} 3$ : $1.2 \% \mathrm{~K} 2 \mathrm{P}$ for $A$. gigas and $0.3 \% \mathrm{~K} 2 \mathrm{P}$ for $P$. soyoae; $H=0.88 \pm 0.0002$ $S D)$. Based on the nuclear genes, the $A$. gigas and $P$. soyoae populations were weakly to highly differentiated $\left(F_{\mathrm{ST}}\right.$ : 0.077-0.321), while usually no differentiation was present within species (Appendix S5). The symbiont $16 \mathrm{~S}$ rRNA gene mirrored the two host nuclear genes in terms of clade differentiation (0.42\% K2P distance), but showed a lower diversity (A. gigas: 0.3\% K2P; P. soyoae: 0.1\% K2P; 11 phylotypes; $H=0.77 \pm 0.021 S D$ ).

In 15 individuals, the $P$. soyoae-specific symbiont $16 S$ rRNA phylotype was found in clams that had the A. gigas mitotype. We observed 13 of these discrepancies at Pedro's Whalefall, where A. gigas was the only clam species found. The two other discrepancies were observed at Extrovert Cliff and Ben's Seep, where both species co-occurred. In all other sequenced clams, the symbiont 165 rRNA lineages corresponded to the host $\mathrm{mtCO}$ lineages, as expected for symbioses with vertical transmission (Table 2).

\section{2 | RADseq single nucleotide polymorphisms: Genomic divergence between clam species}

Genotype likelihood estimations in ANGSD resulted in a total of 349,288 shared sites for population genetic inferences. Principal component analyses based on this data set indicated a clear distinction of three different genetic groups corresponding to pure A. gigas, pure $P$. soyoae and the Pedro's Whalefall clams that contained the A. gigas mitotype but the P. soyoae symbiont phylotype (Figure 3). The first two principal components explained $43.18 \%$ of the variance in this data set. The PCA results were confirmed by admixture analyses that grouped all clam populations as separate entities without any evidence for recent introgression (Appendix S6). On a genome-wide scale, the hybrid and typical $A$. gigas were weakly differentiated $\left(F_{\mathrm{ST}}=0.105\right)$, while both of these groups showed a high divergence from $P$. soyoae $\left(F_{\mathrm{ST}}\right.$ P. soyoae-A. gigas: $0.341 ; F_{\mathrm{ST}} P$. soyoae-hybrid A. gigas: 0.328 ).

\section{3 | Gene flow}

Both $\partial_{A} \partial$ I and IMA2 analyses provided evidence for divergence with gene flow between the two clam species, supporting models of

TABLE 2 Host mitochondrial COI and symbiont 16S rRNA combinations found in this study

\begin{tabular}{|llc|}
\hline Mitotype & Ribotype & N \\
\hline A. gigas & A. gigas & 34 \\
P. soyoae & P. soyoae & 48 \\
\hline A. gigas & P. soyoae & $15^{\mathrm{a}}$ \\
\hline P. soyoae & A. gigas & 0 \\
\hline
\end{tabular}

aPedro's Whalefall: 13, Extrovert Cliff: 1, Ben's Seep: 1. asymmetric migration from $P$. soyoae into A. gigas (Figures 4 and 5; Table 3). Despite the large phylogenetic distance between the two clam genera, the IMA2 analyses could not approximate the time of population splitting or ancestral population size accurately, which indicates a lack of information to constrain these parameters due to the limited number of loci examined. $\partial_{A} \partial_{1}$ favoured the secondary contact model with heterogeneous gene flow (SC2M) as most likely scenario of the speciation process (Table 3; AIC weight for SC2M: 1.00; AIC weights for other models: 0.00). This model suggested a recent secondary contact event after a comparatively long time of species divergence, resulting in (a) mainly neutral gene flow into A. gigas and (b) reduced migration of barrier genes between species. While the SC2M model fits the data significantly better than any other model, all models with two classes of gene flow parameters (IM2M and AM2M) were better fits to the data than those without (Table 3), and better predicted the AFS observed in A. gigas and $P$. soyoae (Figure 5), suggesting that accounting for differential introgression rates across the genome is useful to predict certain characteristics of our data.

\section{4 | DISCUSSION}

Obligately vertical transmission results in co-inheritance of symbionts with the mitochondrial genome of the host. Under this scenario, genetic coupling and ultimately cospeciation of host and symbiont lineages are expected, unless symbionts are occasionally transferred between host species (reviewed in Vrijenhoek, 2010). In

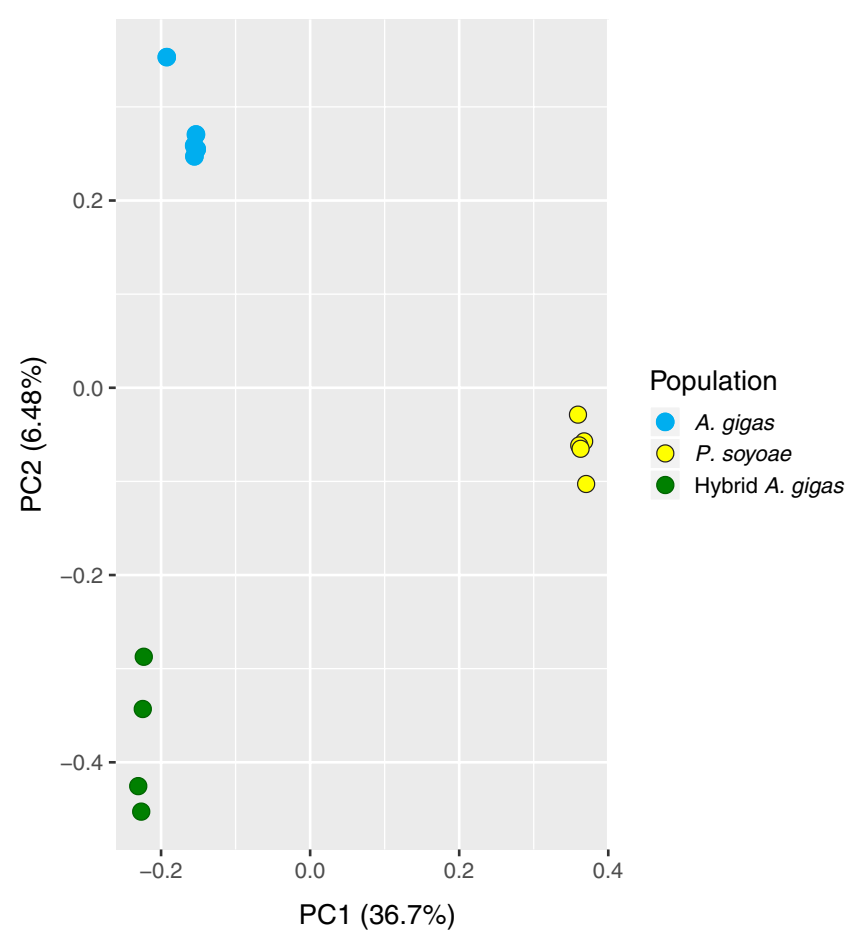

FIGURE 3 PCA plot based on the genome-wide RADseq data set. The hybrid A. gigas-like clams (green) form a separate group from the pure species (yellow, blue) 
FIGURE 4 Isolation-with-migration analyses. (a) Migration rates indicating significant gene flow from $P$. soyoae to A. gigas in the evolutionary past; (b) effective population sizes; (c) time of population splitting

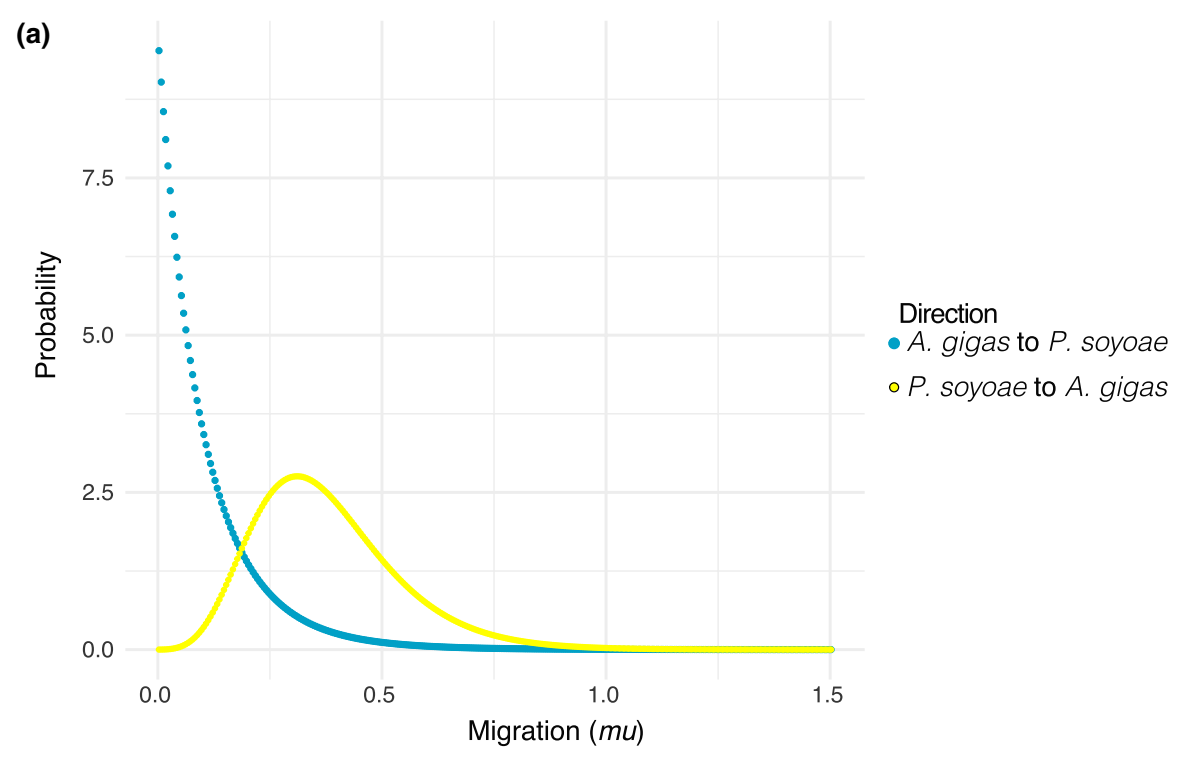

(b)

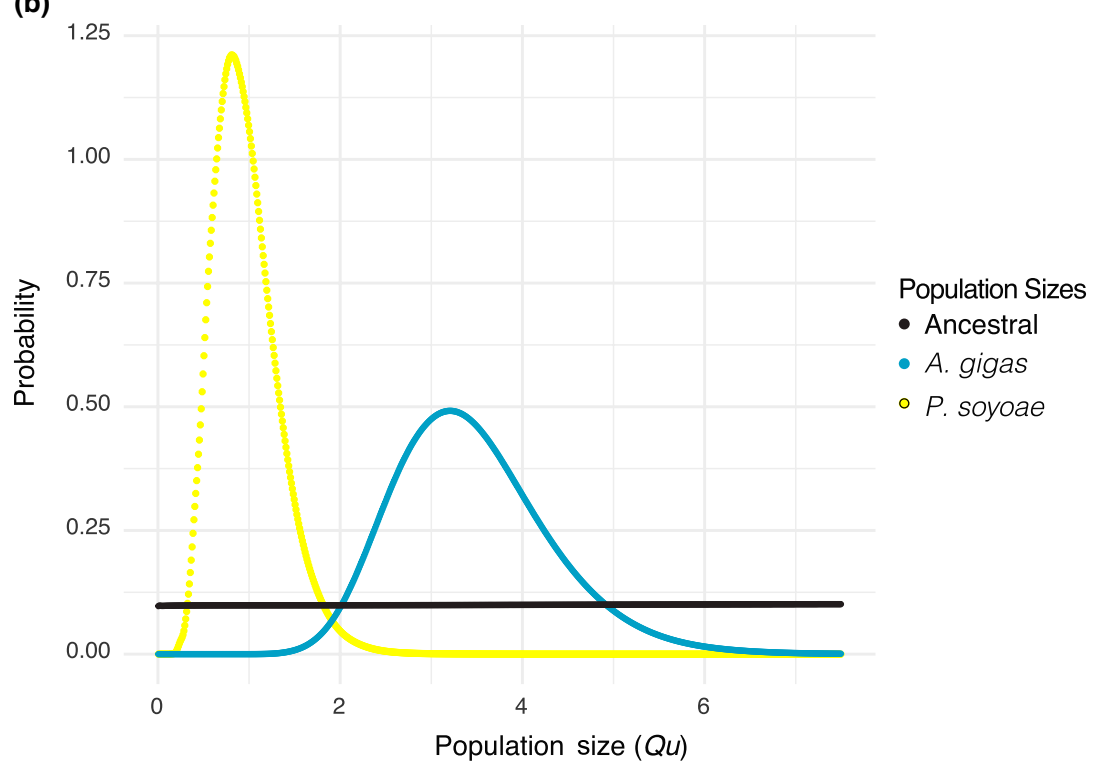

(c)

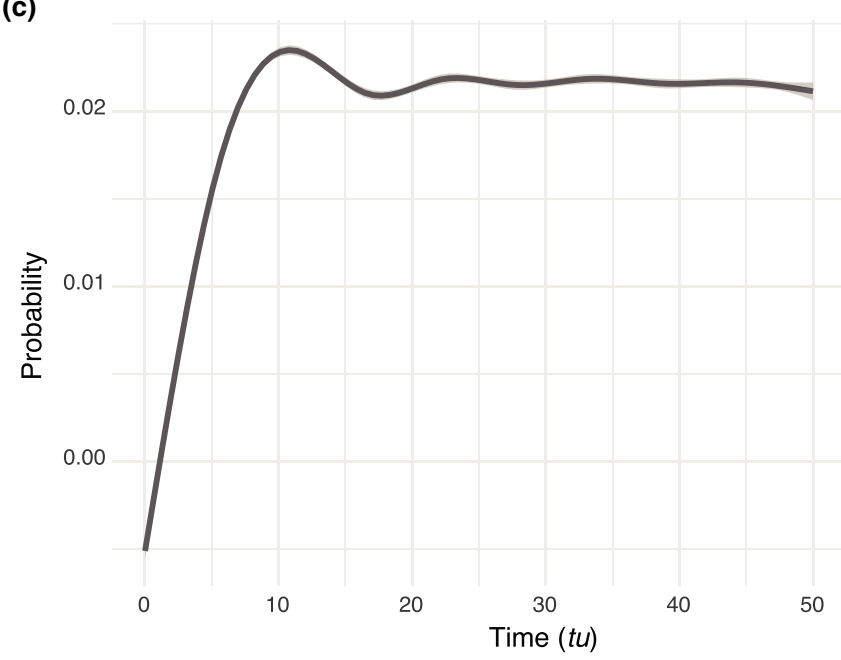

vesicomyid clams, several instances of symbiont leakage have been reported by previous studies (Decker et al., 2013; Ozawa et al., 2017; Stewart \& Cavanaugh, 2009; Stewart et al., 2008; Stewart, Young,
\& Cavanaugh, 2009), but the underlying mechanisms remain poorly understood. Stewart et al. (2008) suggested three different circumstances under which lateral symbiont transfer could occur: (a) host 

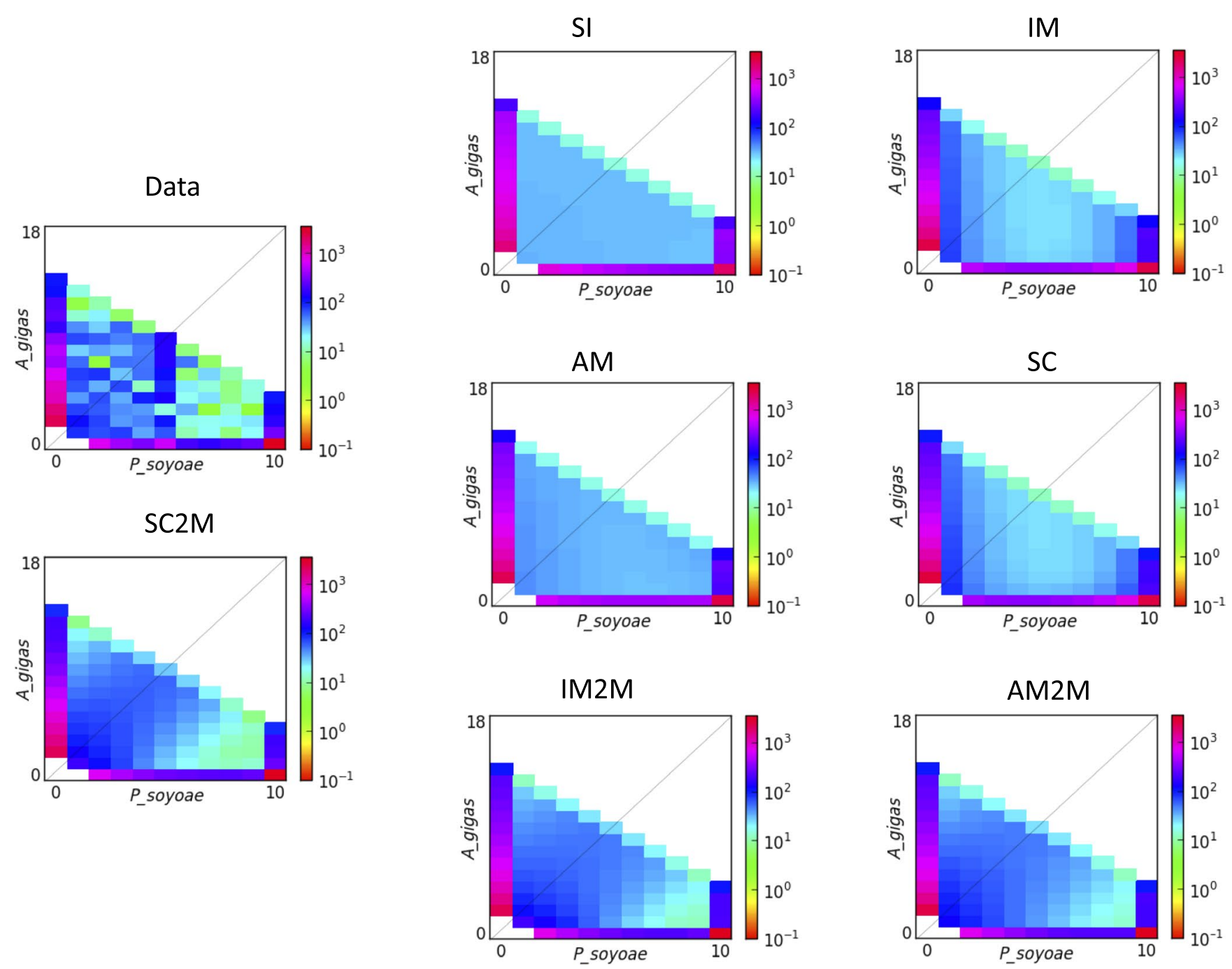

FIGURE 5 Observed and fitted joint folded allele frequency spectra as calculated in $\partial_{A} \partial_{\text {I. }}$. The figure shows the AFS of A. gigas ( $x$-axis, nine individuals) plotted against the AFS of $P$. soyoae ( $y$-axis, five individuals). The colour scheme indicates the frequencies of minor alleles in each population across all polymorphic sites. The SC2M model was the scenario with the highest likelihood, and its AFS is shown in comparison with the other tested models

hybridization, (b) environmental acquisition from a free-living symbiont population or (c) host-to-host transfer, for example through direct contact between symbiont-bearing eggs or uptake of symbiont cells that have been released from a dying clam individual. Although the host-to-host transfer hypothesis has been favoured by multiple authors (Decker et al., 2013; Ikuta et al., 2016; Ozawa et al., 2017; Stewart et al., 2008), the possibility of interspecific hybridization has never been investigated.

Hybridization between different species is an important evolutionary process that can provide fundamental insights into the molecular mechanisms of reproductive isolation and adaptation. The outcomes of interspecific gene flow can be seen as a continuum of two extremes: erosion of species barriers through merging of gene pools (Allendorf, Leary, Spruell, \& Wenburg, 2001) or evolution of new species through novel adaptive trait combinations in hybrids (Gompert, Fordyce, Forister, Shapiro, \& Nice, 2006; Marques, Meier, \& Seehausen, 2019; Seehausen, 2004, 2013). Recent studies have emphasized the importance of symbiotic microbes in animal speciation, with particular focus on their roles in hybrid incompatibility and reinforcement of existing species boundaries (Brucker \& Bordenstein, 2012). While this emerging concept highlights the interactions between hybridization and symbiosis in evolution, synergistic effects of these two mechanisms in adaptive trait introgression and hybrid speciation are unknown.

In this study, we examined the symbiont composition and host hybridization hypothesis in the two clam species, A. gigas and $P$. soyoae, from cold seep sites in the Pacific Ocean. Our barcoding analyses indicated that several individuals with the A. gigas mitotype contained the $P$. soyoae-specific symbiont phylotype at localities where both host species either co-occurred (Ben's Seep and Extrovert Cliff) or where A. gigas was the only taxon found (Pedro's Whalefall). Demographic inference provided evidence that asymmetric gene flow between $P$. soyoae and $A$. gigas did occur in the evolutionary history of the two species. Both IMA 2 and $\partial_{A} \partial$ I analyses 


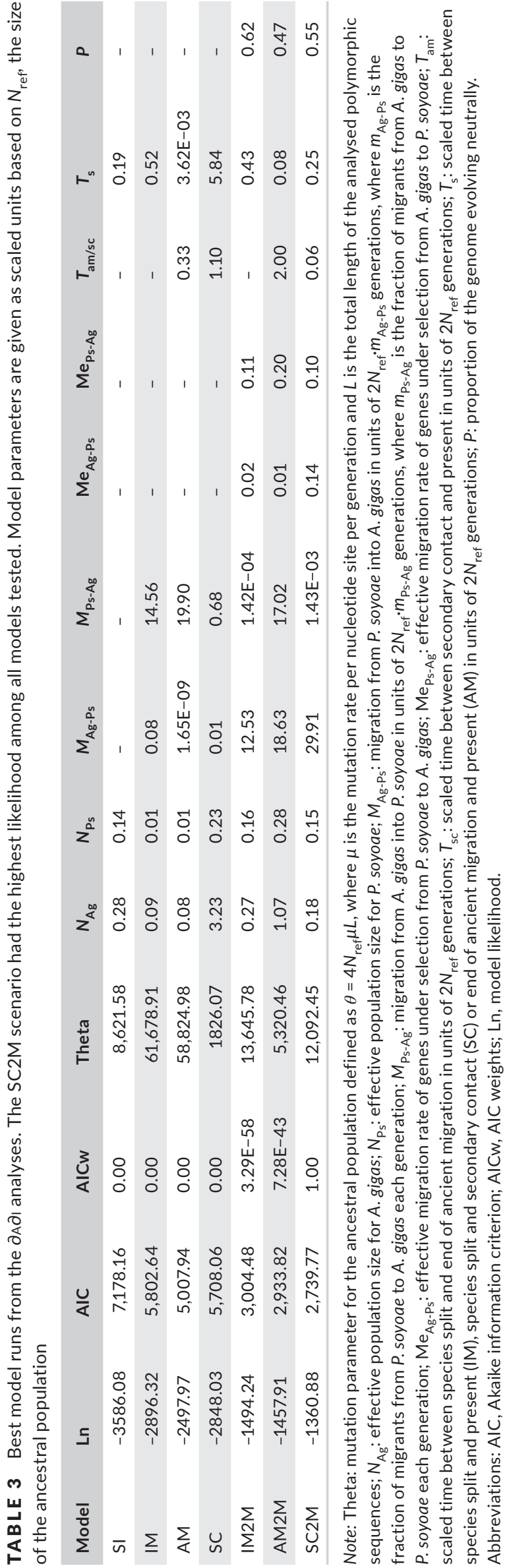

favoured a model of mostly unidirectional migration from $P$. soyoae to $A$. gigas over models of strict isolation. Based on genomic information, $\partial_{A} \partial$ I identified a secondary contact scenario with differential gene introgression as best fit to the observed joint allele frequency spectrum. The SC2M model is the most likely demographic scenario, among those that we examined, underlying the evolutionary divergence of these clam species. Interestingly, however, we did not find any evidence for admixed individuals in the investigated clam populations, as might be expected under a recent secondary contact scenario. This observation could be due to insufficient sampling. It is also possible that there have been enough generations that the genetic disequilibria from a recent hybridization event have nearly reached equilibrium.

Although open questions about the demographic history of $A$. gigas and $P$. soyoae remain, our data suggest that interspecific hybridization could be a mechanism for horizontal symbiont transmission and might explain occurrences of the $P$. soyoae symbiont phylotype in A. gigas-like clam hosts. Surprisingly, we did not observe the alternative combination, that is $P$. soyoae hosts with the $A$. gigas symbiont. A simple technical explanation for this phenomenon could be sampling gaps, which are notorious problems in deep-sea research. A possible biological explanation for our findings that is in line with the outputs of the SC2M model is asymmetric genetic incompatibilities between host species and symbionts that result in strong selection against the $P$. soyoae host $\times A$. gigas symbiont combination. Vertical transmission usually leads to co-adaptation of host-symbiont gene complexes, which are often disrupted through interspecific hybridization (Bordenstein, O'Hara, \& Werren, 2001; Bordenstein \& Werren, 2007; Brucker, \& Bordenstein, 2012, 2013; Jaenike, Dyer, Cornish, \& Minhas, 2006; Vala, Breeuwer, \& Sabelis, 2000). Under this scenario, the $P$. soyoae symbiont would have an unknown fitness advantage that favours switching to a new host.

Incongruent compositions of host mitochondrial and symbiont genomes due to hybridization must involve some form of paternal cotransmission. One possibility is occasional inheritance of mitochondria and symbionts through the paternal germline (paternal leakage). Paternal leakage is a common phenomenon that occurs in a variety of different taxa at low frequency (reviewed in Breton \& Stewart, 2015), which would agree with our observation that introgressed symbionts are rare. The second possibility is doubly uniparental inheritance (DUI) of mitochondria, which would involve a regulated system of paternal cotransmission as suggested previously for Vesicomya sp. mt-II (Stewart et al., 2008). Although DUI has not been described in vesicomyid clams, it is known to occur in some Veneroida (Gusman, Lecomte, Stewart, Passamonti, \& Breton, 2016; Zouros, 2013). To determine whether DUI is present in vesicomyids, it would be necessary to sequence mitochondrial genomes from sexed individuals and identify if genetic differences exist between female and male mitochondria.

Despite supporting hybridization as a potential mechanism of lateral symbiont transfer, our data do not reject the alternative hypotheses that were stated in the literature, although those seem less likely in this case for several reasons. First, the symbionts of $A$. gigas 
and $P$. soyoae belong to vesicomyid symbiont Clade I which is characterized by highly reduced genomes without essential genes for an extracellular lifestyle (Kuwahara et al., 2011), so that it is improbable that a free-living population of these symbionts exists in the environment. Second, if physical proximity promoted lateral symbiont acquisition as argued by Decker et al. (2013), we would expect to find more individuals with non-native symbionts in sympatric populations of $A$. gigas and $P$. soyoae. Furthermore, non-native symbiont phylotypes were only observed in A. gigas, but not in $P$. soyoae. If host-to-host transfer was the underlying mechanism leading to symbiont switching, foreign phylotypes should be observed in both species, unless natural selection acted against the $P$. soyoae host $\times$ A. gigas symbiont combination (as mentioned above).

Our results raise several interesting hypotheses that can be addressed in future studies. In this study, we only investigated the symbiont $16 \mathrm{~S}$ rRNA gene. To disentangle host-to-host transfer from retention of an introgressed strain, it will be necessary to sequence and compare whole genomes of symbionts from clam individuals that contain the native and foreign $P$. soyoae phylotypes. A symbiont that was transferred historically via host hybridization can be expected to be highly divergent from the native phylotype, while a symbiont that was transmitted via contemporary host-to-host transfer should be relatively similar. Since we sequenced the $16 \mathrm{~S}$ rRNA gene directly, we were not able to uncover potential symbiont mixtures in individual host animals, given that this sequencing approach is biased towards the most abundant phylotype (Zimmermann et al., 2014). Furthermore, symbiont types could be variable across the host gill, as seen in other taxa from chemosynthetic environments (Duperron et al., 2005; Zimmermann et al., 2014). Studies that involved cloning and pyrosequencing techniques showed that in some cases divergent symbiont lineages can co-occur in a single clam host (Decker et al., 2013; Stewart \& Cavanaugh, 2009). To examine whether and how different symbiont lineages coexist in A. gigas and P. soyoae, whole-genome analyses based on high-throughput sequencing techniques will be useful. Comparative genomic and population genomic approaches will help to illuminate the genomic consequences of occasional lateral symbiont acquisition in deep-sea vesicomyid clams and lead to a better understanding of how host-symbiont interactions shape the evolution of both partners.

\section{ACKNOWLEDGEMENTS}

We thank the crews of the RV Point Lobos, Rachel Carson and Western Flyer and pilots of the ROV Ventana, Tiburon and Doc Ricketts for carefully collecting innumerable clams. We would also like to thank Jim Barry for letting us raid his freezers to collect even more clams. Thank you as well to Shana Goffredi who was responsible for many of the early clam collections and also provided useful discussions about symbiosis. We also want to thank Brian Dalley who developed and tested the ezRAD library preparation protocol for us. Funding was provided by grants of the German Research Foundation (BR 5488/1-1 to C.B.), the David and Lucile Packard Foundation (to MBARI) and the National Science Foundation
(OCE9302205, OCE0241613 to R.C.V.). C.R.Y. received support from the UK Natural Environment Research Council through the MARINe-DNA Project (grant number NE/N006496/1) and National Capability funding to the National Oceanography Centre as part of the Climate Linked Atlantic Section Science (CLASS) programme (grant number NE/R015953/1).

\section{AUTHOR CONTRIBUTIONS}

C.B. and S.B.J. designed the study. C.B. performed the RADseq, bioinformatic and statistical analyses and wrote the manuscript. S.B.J. found the mismatched symbiont strains by Sanger sequencing, produced the graphics and conducted the statistical analyses for the Sanger sequenced genes. C.R.Y. and R.C.V. advised on the study concept and statistical analyses. All authors contributed to writing the manuscript and agreed to this version of the manuscript.

\section{DATA AVAILABILITY STATEMENT}

Sanger sequenced reads for $16 \mathrm{~S}, \mathrm{mtCO}, \mathrm{H} 3$ and ANT have been uploaded to GenBank (https://www.ncbi.nlm.nih.gov/genbank/) under Accession nos MK060220-MK060689. Raw ezRAD data have been deposited in the Sequence Read Archive (https://www. ncbi.nlm.nih.gov/sra) under BioProject number PRJNA497587. Bioinformatic code for the ezRAD analyses is provided in the Appendix S1-S6.

\section{ORCID}

Corinna Breusing (iD https://orcid.org/0000-0001-6845-0188

\section{REFERENCES}

Allendorf, F. W., Leary, R. F., Spruell, P., \& Wenburg, J. K. (2001). The problems with hybrids: Setting conservation guidelines. Trends in Ecology and Evolution, 16, 613-622. https://doi.org/10.1016/ S0169-5347(01)02290-X

Archibald, J. M. (2015). Endosymbiosis and eukaryotic cell evolution. Current Biology, 25, R911-921. https://doi.org/10.1016/j. cub.2015.07.055

Benjamini, Y., \& Yekutieli, D. (2001). The control of the false discovery rate in multiple testing under dependency. Annals of Statistics, 29, 1165-1188.

Bennett, G. M., \& Moran, N. A. (2015). Heritable symbiosis: The advantages and perils of an evolutionary rabbit hole. Proceedings of the National Academy of Sciences of the USA, 112, 10169-10176. https:// doi.org/10.1073/pnas.1421388112

Bolger, A. M., Lohse, M., \& Usadel, B. (2014). Trimmomatic: A flexible trimmer for Illumina sequence data. Bioinformatics, 30, 2114-2120. https://doi.org/10.1093/bioinformatics/btu170

Bordenstein, S. R., O'Hara, F. P., \& Werren, J. H. (2001). Wolbachia-induced incompatibility precedes other hybrid incompatibilities in Nasonia. Nature, 409, 707-710. https://doi.org/10.1038/35055543

Bordenstein, S. R., \& Werren, J. H. (2007). Bidirectional incompatibility among divergent Wolbachia and incompatibility level differences 
among closely related Wolbachia in Nasonia. Heredity, 99, 278-287. https://doi.org/10.1038/sj.hdy.6800994

Breton, S., \& Stewart, D. T. (2015). Atypical mitochondrial inheritance patterns in eukaryotes. Genome, 58, 423-431. https://doi. org/10.1139/gen-2015-0090

Breusing, C., Johnson, S. B., Tunnicliffe, V., \& Vrijenhoek, R. C. (2015). Population structure and connectivity in Indo-Pacific deep-sea mussels of the Bathymodiolus septemdierum complex. Conservation Genetics, 16, 1415-1430. https://doi.org/10.1007/ s10592-015-0750-0

Bright, M., \& Bulgheresi, S. (2013). A complex journey: Transmission of microbial symbionts. Nature Reviews Microbiology, 8, 218-230. https ://doi.org/10.1038/nrmicro2262

Brucker, R. M., \& Bordenstein, S. R. (2012). Speciation by symbiosis. Trends in Ecology and Evolution, 27, 443-451. https://doi. org/10.1016/j.tree.2012.03.011

Brucker, R. M., \& Bordenstein, S. R. (2013). The hologenomic basis of speciation: Gut bacteria cause hybrid lethality in the genus Nasonia. Science, 341, 667-669. https://doi.org/10.1126/science.1240659

Burnham, K. P., \& Anderson, D. R. (2002). Model selection and multi model inference. A practical information-theoretic approach (488 pp). New York, NY: Springer.

Decker, C., Olu, K., Arnaud-Haond, S., \& Duperron, S. (2013). Physical proximity may promote lateral acquisition of bacterial symbionts in vesicomyid clams. PLoS ONE, 8, e64830. https://doi.org/10.1371/ journal.pone.0064830

Dubilier, N., Bergin, C., \& Lott, C. (2008). Symbiotic diversity in marine animals: The art of harnessing chemosynthesis. Nature Reviews Microbiology, 6, 725-740. https://doi.org/10.1038/nrmicro1992

Duperron, S., Nadalig, T., Caprais, J. C., Sibuet, M., Fiala-Médioni, A., Amann, R., \& Dubilier, N. (2005). Dual symbiosis in a Bathymodiolus sp. mussel from a methane seep on the Gabon continental margin (Southeast Atlantic): 16S rRNA phylogeny and distribution of the symbionts in gills. Applied and Environmental Microbiology, 71, 1694-1700.

Ebert, D. (2013). The epidemiology and evolution of symbionts with mixed-mode transmission. Annual Review of Ecology, Evolution, and Systematics, 44, 623-643. https://doi.org/10.1146/annurev-ecols ys-032513-100555

Fumagalli, M., Vieira, F. G., Linderoth, T., \& Nielsen, R. (2014). ngsTools: Methods for population genetics analyses from next-generation sequencing data. Bioinformatics, 30, 1486-1487. https://doi. org/10.1093/bioinformatics/btu041

Gompert, Z., Fordyce, J. A., Forister, M. L., Shapiro, A. M., \& Nice, C. C. (2006). Homoploid hybrid speciation in an extreme habitat. Science, 314, 1923-1925. https://doi.org/10.1126/science.1135875

Gusman, A., Lecomte, S., Stewart, D. T., Passamonti, M., \& Breton, S. (2016). Pursuing the quest for better understanding the taxonomic distribution of the system of doubly uniparental inheritance of mtDNA. PeerJ, 4, e2760. https://doi.org/10.7717/peerj.2760

Gutenkunst, R. N., Hernandez, R. D., Williamson, S. H., \& Bustamante, C. D. (2009). Inferring the joint demographic history of multiple populations from multidimensional SNP data. PLoS Genetics, 5, e1000695.

Hey, J. (2010). Isolation with migration models for more than two populations. Molecular Biology and Evolution, 27, 905-920. https://doi. org $/ 10.1093 / \mathrm{molbev} / \mathrm{msp} 296$

Ikuta, T., Igawa, K., Tame, A., Kuroiwa, T., Kuroiwa, H., Aoki, Y., ... Yoshida, T. (2016). Surfing the vegetal pole in a small population: Extracellular vertical transmission of an 'intracellular' deep-sea clam symbiont. Royal Society Open Science, 3, 160130. https://doi.org/10.1098/ rsos.160130

Jaenike, J., Dyer, K. A., Cornish, C., \& Minhas, M. S. (2006). Asymmetrical reinforcement and Wolbachia infection in Drosophila. PLoS Biology, 4, e325. https://doi.org/10.1371/journal.pbio.0040325

Johnson, S. B., Krylova, E. M., Audzijonyte, A., Sahling, H., \& Vrijenhoek, R. C. (2016). Phylogeny and origins of chemosynthetic vesicomyid clams. Systematics and Biodiversity, 15, 346-360. https:// doi.org/10.1080/14772000.2016.1252438

Korneliussen, T. S., Albrechtsen, A., \& Nielsen, R. (2014). ANGSD: Analysis of next generation sequencing data. BMC Bioinformatics, 15, 356. https://doi.org/10.1186/s12859-014-0356-4

Kumar, S., Stecher, G., Li, M., Knyaz, C., \& Tamura, K. (2018). MEGA X: Molecular evolutionary genetics analysis across computing platforms. Molecular Biology and Evolution, 35, 1547-1549. https://doi. org $/ 10.1093 / \mathrm{molbev} / \mathrm{msy} 096$

Kuwahara, H., Takaki, Y., Shimamura, S., Yoshida, T., Maeda, T., Kunieda, T., ... Maruyama, T. (2011). Loss of genes for DNA recombination and repair in the reductive genome evolution of thioautotrophic symbionts of Calyptogena clams. BMC Evolutionary Biology, 11, 285. https:// doi.org/10.1186/1471-2148-11-285

Kuwahara, H., Yoshida, T., Takaki, Y., Shimamura, S., Nishi, S., Harada, M., ... Maruyama, T. (2007). Reduced genome of the thioautotrophic intracellular symbiont in a deep-sea clam, Calyptogena okutanii. Current Biology, 17, 881-886. https://doi.org/10.1016/j.cub.2007.04.039

Lane, D. J. (1991). 16S/23S rRNA sequencing. In E. Stackebrandt, \& M. Goodfellow (Eds.), Nucleic acid techniques in bacterial systematics (pp. 115-147). New York, NY: John Wiley \& Sons.

Marques, D. A., Meier, J. I., \& Seehausen, O. (2019). A combinatorial view on speciation and adaptive radiation. Trends in Ecology and Evolution, 34, 531-544. https://doi.org/10.1016/j.tree.2019.02.008

McFall-Ngai, M. (2008). Are biologists in 'future shock'? Symbiosis integrates biology across domains. Nature Reviews Microbiology, 6, 789792. https://doi.org/10.1038/nrmicro1982

McFall-Ngai, M., Hadfield, M. G., Bosch, T. C. G., Carey, H. V., DomazetLošo, T., Douglas, A. E., ... Wernegreen, J. J. (2013). Animals in a bacterial world, a new imperative for the life sciences. Proceedings of the National Academy of Sciences of the USA, 110, 3229-3236. https://doi. org/10.1073/pnas.1218525110

Meirmans, P. G., \& van Tienderen, P. H. (2004). GENOTYPE and GENODIVE: Two programs for the analysis of genetic diversity of asexual organisms. Molecular Ecology Resources, 4, 792-794.

Moran, N. A., \& Dunbar, H. E. (2006). Sexual acquisition of beneficial symbionts in aphids. Proceedings of the National Academy of Sciences of the USA, 103, 12803-12806. https://doi.org/10.1073/pnas.06057 72103

Moran, N. A., McCutcheon, J. P., \& Nakabachi, A. (2008). Genomics and evolution of heritable bacterial symbionts. Annual Review of Genetics, 42, 165-190. https://doi.org/10.1146/annur ev.genet.41.110306.130119

Ozawa, G., Shimamura, S., Takaki, Y., Takishita, K., Ikuta, T., Barry, J. P., ... Yoshida, T. (2017). Ancient occasional host switching of maternally transmitted bacterial symbionts of chemosynthetic vesicomyid clams. Genome Biology and Evolution, 9, 2226-2236. https://doi. org/10.1093/gbe/evx166

Puritz, J. B., Hollenbeck, C. M., \& Gold, J. R. (2014). dDocent: A RADseq, variant-calling pipeline designed for population genomics of nonmodel organisms. PeerJ, 2, e431.

Rispe, C., \& Moran, N. A. (2000). Accumulation of deleterious mutations in endosymbionts: Muller's Ratchet with two levels of selection. The American Naturalist, 156, 425-441. https://doi.org/10.1086/303396

Seehausen, O. (2004). Hybridization and adaptive radiation. Trends in Ecology and Evolution, 19, 198-207. https://doi.org/10.1016/j. tree.2004.01.003

Seehausen, O. (2013). Conditions when hybridization might predispose populations for adaptive radiation. Journal of Evolutionary Biology, 26, 279-281. https://doi.org/10.1111/jeb.12026

Shimamura, S., Kaneko, T., Ozawa, G., Matsumoto, M. N., Koshiishi, T., Takaki, Y., ... Maruyama, T. (2017). Loss of genes related to Nucleotide Excision Repair (NER) and implications for reductive genome evolution in symbionts of deep-sea vesicomyid clams. PLOS ONE, 12, e0171274. https://doi.org/10.1371/journal.pone.0171274 
Sloan, D. B., \& Moran, N. A. (2012). Genome reduction and co-evolution between the primary and secondary bacterial symbionts of psyllids. Molecular Biology and Evolution, 29, 3781-3792. https://doi. org/10.1093/molbev/mss180

Stewart, F. J., \& Cavanaugh, C. M. (2009). Pyrosequencing analysis of endosymbiont population structure: Co-occurrence of divergent symbiont lineages in a single vesicomyid host clam. Environmental Microbiology, 11, 2136-2147. https://doi.org/10.1111/j.1462-2920.2009.01933.x

Stewart, F. J., Young, C. R., \& Cavanaugh, C. M. (2008). Lateral symbiont acquisition in a maternally transmitted chemosynthetic clam endosymbiosis. Molecular Biology and Evolution, 25, 673-687. https://doi. org/10.1093/molbev/msn010

Stewart, F. J., Young, C. R., \& Cavanaugh, C. M. (2009). Evidence for homologous recombination in intracellular chemosynthetic clam symbionts. Molecular Biology and Evolution, 26, 1391-1404. https://doi. org/10.1093/molbev/msp049

Strasburg, J. L., \& Rieseberg, L. H. (2010). How robust are "isolation with migration" analyses to violations of the IM model? A simulation study. Molecular Biology and Evolution, 27, 297-310. https://doi. org/10.1093/molbev/msp233

Tine, M., Kuhl, H., Gagnaire, P.-A., Louro, B., Desmarais, E., Martins, R. S. T., ... Reinhardt, R. (2014). European sea bass genome and its variation provide insights into adaptation to euryhalinity and speciation. Nature Communications, 5, 5770. https://doi.org/10.1038/ncomm s6770

Toonen, R. J., Puritz, J. B., Forsman, Z. H., Whitney, J. L., Fernandez-Silva, I., Andrews, K. R., \& Bird, C. E. (2013). ezRAD: A simplified method for genomic genotyping in non-model organisms. PeerJ, 1, e203. https://doi.org/10.7717/peerj.203

Vala, F., Breeuwer, J. A., \& Sabelis, M. W. (2000). Wolbachia-induced 'hybrid breakdown' in the two-spotted spider mite Tetranychus urticae Koch. Proceedings of the Biological Society of London B: Biological Sciences, 267, 1931-1937.

Vrijenhoek, R. C. (2010). Genetics and evolution of deep-sea chemosynthetic bacteria and their invertebrate hosts. In S. Kiel (Ed.), The Vent and Seep Biota (pp. 15-49). Dordrecht, Netherlands: Springer.

Vrijenhoek, R. C., Duhaime, M., \& Jones, W. J. (2007). Subtype variation among bacterial endosymbionts of tubeworms (Annelida:
Siboglinidae) from the Gulf of California. The Biological Bulletin, 212 180-184. https://doi.org/10.2307/25066600

Warmuth, V. M., \& Ellegren, H. (2019). Genotype-free estimation of allele frequencies reduces bias and improves demographic inference from RADSeq data. Molecular Ecology Resources, 19(3), 586-596. https:// doi.org/10.1111/1755-0998.12990

Watanabe, K., Yukuhiro, F., Matsuura, Y., Fukatsu, T., \& Noda, H. (2014). Intrasperm vertical symbiont transmission. Proceedings of the National Academy of Sciences of the USA, 111, 7433-7437. https://doi. org/10.1073/pnas.1402476111

Woerner, A. E., Cox, M. P., \& Hammer, M. F. (2007). Recombination-filtered genomic datasets by information maximization. Bioinformatics, 23, 1851-1853. https://doi.org/10.1093/bioinformatics/btm253

Zimmermann, J., Lott, C., Weber, M., Ramette, A., Bright, M., Dubilier N., \& Petersen, J. M. (2014). Dual symbiosis with co-occurring sulfur-oxidizing symbionts in vestimentiferan tubeworms from a Mediterranean hydrothermal vent. Environmental Microbiology, 16 3638-3656. https://doi.org/10.1111/1462-2920.12427

Zouros, E. (2013). Biparental inheritance through uniparental transmission: The doubly uniparental inheritance (DUI) of mitochondrial DNA. Evolutionary Biology, 40, 1-31. https://doi.org/10.1007/ s11692-012-9195-2

\section{SUPPORTING INFORMATION}

Additional supporting information may be found online in the Supporting Information section at the end of the article.

How to cite this article: Breusing C, Johnson SB, Vrijenhoek RC, Young CR. Host hybridization as a potential mechanism of lateral symbiont transfer in deep-sea vesicomyid clams. Mol Ecol. 2019;28:4697-4708. https://doi.org/10.1111/mec.15224 